\title{
A Study on the Theoretical Model of Port Safety Resilience under Major Emergencies and Its Quantitative Evaluation
}

\author{
Yanhua $\mathrm{Hu}^{1, \mathrm{a}}$, Guodong Meng ${ }^{2, \mathrm{~b}}$, Jianyu $\mathrm{Lv}^{3, \mathrm{c} *}$, Hang Jin ${ }^{4, \mathrm{~d}}$, Linlin $\mathrm{Lu}^{5, \mathrm{e}}$ \\ ${ }^{1}$ Tianjin Research Institute for Water Transport Engineering, M.O.T.Tianjin, China \\ ${ }^{2}$ Tianjin Dongfang Tairui Technology CO. LTD.Tianjin, China \\ ${ }^{3}$ Tianjin Research Institute for Water Transport Engineering, M.O.T.Tianjin, China \\ ${ }^{4}$ Tianjin Research Institute for Water Transport Engineering, M.O.T.Tianjin, China \\ ${ }^{5}$ Tianjin Research Institute for Water Transport Engineering, M.O.T.Tianjin, China
}

\begin{abstract}
With the rapid development of urbanization, China's security system in ports is facing many problems and challenges, especially after the outbreak of COVID-19 has made a great impact on China. In order to avoid the serious losses caused by safety problems in ports under major emergencies due to the lack of necessary, forward-looking prevention in the increasingly complex system of ports, this paper introduced the theory of safety resilience into the field of ports for the first time and proposed a triangle model of safety resilience in ports, which included three elements: the disaster system, the disaster bearing system and the safety resilience management. Aiming at the quantitative evaluation demands of safety resilience in ports, the system evaluation indexes of safety resilience in ports were established, including 6 secondary indexes and 37 tertiary indexes. The theory of entropy weight based on normalization standard processing was used to calculate and analyze the importance of each index, and the influence weight value of each index on safety resilience and the prediction curve chart of safety resilience in ports were obtained. The research results can provide a reference for the capacity construction and application of safety resilience in ports, which will effectively solve the new challenges faced by port safety at this stage and help to improve the ability of the port not only in disaster prevention and mitigation but also in disaster recovery and continuous improvement.
\end{abstract}

\section{Introduction}

Recently, the outbreak of novel coronavirus pneumonia (hereinafter referred to as COVID-19) has greatly affected China's economic development, safe production, transportation and people's life. As an important part of the domestic comprehensive transportation system [1-2], the port is the front line of 'preventing foreign inputs and internal spread in this pandemic, shouldering the industry mission of resolutely blocking the channels for the transmission of the virus, ensuring the smooth operation of the life channels of strategic materials and guaranteeing the continuous performance of the green channels of emergency transportation. When a major public event occurs such as the outbreak of the epidemic, it is easy to deteriorate into a trans-regional outbreak and diffusion of the land-water integrated transport network through ports. In this context, how to effectively deal with the epidemic, realize the safety resilience management of the ports under major emergencies, and maximize the protection of people's lives and property safety and social stability, has become a hot problem to be solved in the industry of ports.

Safety resilience is the frontier concept of current science of public safety, and it has been widely focused on by scholars in China and abroad, especially in the practice

a huyanhua@tk-aq.com, ${ }^{\mathrm{b}}$ mengguodong@tk-aq.com

c* Corresponding author: lvjianyu@tk-aq.com

djinhang@tk-aq.com, elulinlin@tk-aq.com of urban safety management [3-10]. At present, the domestic safety management of ports mainly focuses on the safety production of dangerous goods in the ports [11], the safety protection of equipment and facilities in the ports [12], the safety evaluation and regional risk assessment [13][14] and so on. The connotation analysis of safety resilience in ports has not been carried out, nor is there any theoretical or applied research.

In view of this situation, this paper analyzed the concept of safety resilience, and extends it in the field of port safety, deeply analyzed the demands for safety resilience of domestic ports in response to major emergencies and discussed the establishment of the development model of safety resilience in ports based on major emergencies [15][16]. Combined with the construction of the index system of safety resilience in ports and the calculation and analysis of entropy weights, the influence weight value of each index on safety resilience and the prediction curve chart of safety resilience in ports were obtained, which can provide reference for the capacity construction and application research of safety resilience in ports.

The rest of this article is structured as follows. The second part summarizes the research on security and resilience theory of ports. The third part introduces the research and analysis of establishing the index system of 
safety resilience in ports and the calculation of the weight of each index. The fourth part summarizes the conclusion of this study and analyzes the innovation and limitations of this study and future research directions.

\section{Literature Review}

First, confirm that you have the correct template for your paper size. This template has been tailored for output on the US-letter paper size. If you are using A4-sized paper, please close this file and download the file for "MSW_A4_format".

At present, China has greatly improved the level of security in ports through hidden danger investigation and risk control and other modes. However, after a period of stable security, security problems of ports have occurred frequently in recent years, especially in major emergencies.

For this reason, the concept of resilience is introduced into the study of public safety. Resilient cities focus on the ability to absorb external disturbances, as well as the ability to restore the original state or upgrade to a new equilibrium state through learning after the destruction of the urban system [17-18]. Since the situation of global urban security changed significantly in 2010, the concept of 'resilience', an idea of systematic and sustainable development, has begun to attract wide attention [19].

The word resilience comes from Latin, meaning 'return to the original state' [20]. Holling introduced resilience into ecology for application [21]. In the 1980s, relevant experts and scholars used resilience theory to make research in the field of disaster science. In the 1990s, the resilience theory was applied to the field of human ecology [22] and began to be applied to urban studies. With the research and development of resilience theory, relevant experts and scholars proposed three development processes of engineering resilience, ecological resilience and evolutionary resilience [21]. Among them, the engineering resilience pursues a single equilibrium state, the ecological resilience pursues a multiple equilibrium state, and the evolutionary resilience pursues the dynamic ability of rapid recovery and improvement, which reflects the change of resilience theory in academia and makes theoretical and practical preparations for proposing the resilience of safety production management. Hollnagel et al. believed that the traditional safety management cannot adapt to the rapid development of society, and the research of safety science should be transformed to safety resilience so that the system can quickly restore its operation state when it is subjected to external shocks [23]. Righiaw et al. analyzed the application of resilience theory in safety management, accident analysis and risk assessment [24].

Academician Fan et al. pointed out that safety resilience must be emphasized in the system of public security [25]. Huang and $\mathrm{Wu}$ et al. proposed the definition of system safety resilience and constructed the evaluation model of system safety resilience, while carefully analyzing and studying the related theories of system safety resilience [26]. Based on the modeling theory of system science, Luo and Huang et al. believed that the management of safety production in the future should be RBS / M, namely, risk-based management [27]. Li and
Zhai, on the basis of extensive reading of relevant literature on international infrastructure, sorted out and summarized the quantitative evaluation method of urban infrastructure resilience [28].

Desouza et al. [29] constructed a conceptual framework for the design, the planning and the management of the resilient city to assess the adaptability and vulnerability of cities to pressure sources, to understand the interactive mode of relevant urban organizations, to solve the coordination problems among organizations, and to achieve the resilient management and practice long-term management. Jha et al. [30] discussed the principles, tools and practices of building resilient cities, and proposed a method to evaluate a city's resilience by focusing on natural disasters and climate changes. Cutter et al. [31] believed that urban resilience was not only reflected in a single dimension of infrastructure, but also should consider numerous influencing factors of the society, the economy, the organizational system, the infrastructure, the ecology and other aspects to construct a comprehensive index system of urban resilience evaluation. Cutter et al. [32] proposed a relatively complete evaluation index of urban resilience based on previous studies, mainly giving evaluations from the aspects of climate disasters, economy, community organization policies and infrastructure.

In summary, there are few current in-depth studies on the combination of resilience theory and safety production theory in the field of port safety at home and abroad. It is necessary to practice further researches based on these studies, and to systematically study the inherent laws of the resilience management of safety production in ports, so as to put forward new paths and methods for port enterprises to carry out risk and safety management under major emergencies.

\section{Theory of Safety Resilience in Ports and Its Model Construction}

At present, the triangle model of safety resilience is the classical basic theoretical model in the field of public security management [33]. It takes emergency events, bearing carriers and emergency management as three edges, and takes disaster factors (materials, abilities and information) as nodes to connect the three edges, revealing the basic elements of the emergency management of public security. On this basis, this paper proposes a triangle model for the development of safety resilience in ports (as shown in Figure 1). This model takes the disaster system, the disaster bearing system and the safety resilience management as three edges, and connects each edge through such response processes as the planning and construction of the port, the operation and maintenance, the event response and the recovery and reconstruction. In this model, the disaster system includes the sudden incidents of public security (the epidemic, the terrorist attacks, etc.), the natural disasters (the volcanic eruptions, the earthquakes, etc.) and the collection of various disaster-causing energies caused by other irresistible forces. The disaster-bearing system includes the workplace involved in the production and operation of the 
port and all the basic units that may produce risks, cause hidden dangers or lead to accidents in the workplace, and also includes people who have unsafe behavior. The safety resilience management is a systematic and human intervention to the disaster-bearing system in the port when the disaster occurs.

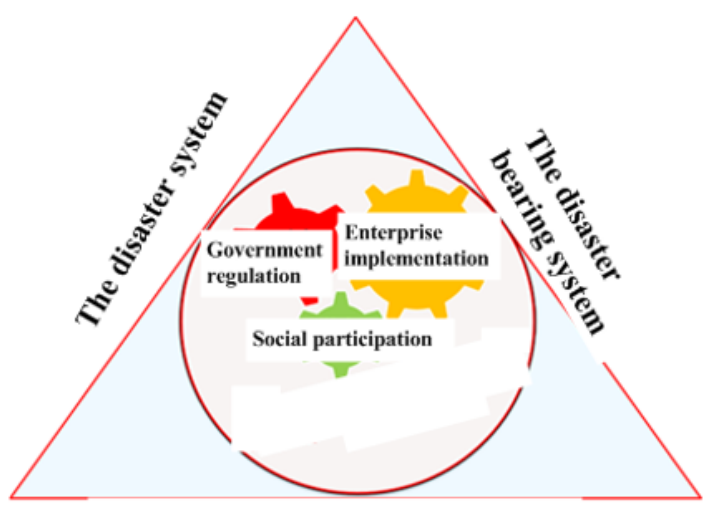

The safety resilience management

Figure 1 The Triangle Model of Safety Resilience in Ports

The triangle model of the development of the safety resilience in ports aims to build a set of redundant system of safety management based on the theory of safety resilience. Through the government supervision, enterprise implementation and social participation, the culture atmosphere of safety fully participated by all members shall be created. At the same time, this system covers the whole process of risk assessment, risk early warning, risk control and hidden danger management "before the accident", the emergency response and the rescue disposal "during the accident", and the recovery and reconstruction, the accident investigation and the analysis and treatment "after the accident". From strengthening the source management, the process control, the basic guarantee, the emergency response and other aspects, the abilities of the disaster bearing system (such as ports, cities, regions, etc.) to identify, evaluate, control and adapt to risks shall be continuously improved, so as to focus on improving the disaster bearing resilience and safety allowance in safe production, scientific operation and emergency management, and ultimately reduce the risk of safe production to an acceptable and reasonable range.

\subsection{Demand Analysis of Safety Resilience in Ports}

Figure 2 describes the port throughput of Dalian port before and after the ' 7.16 ' explosion accident of oil pipelines in 2010. Figure 3 shows the port throughput of Tianjin port before and after the extraordinarily serious ' 8.12 ' fire and explosion accident of the warehouse of the dangerous goods of Rui Hai Corporation in 2015, and Figure 4 illustrates the port throughputs of large-scale national ports before and after the outbreak of the pandemic from April 2019 to April 2020. From Figure 2, Figure 3 and Figure 4, it is not difficult to find that all kinds of serious emergencies of public safety in ports will not only cause huge casualties and property losses, but also have a great impact on the port throughput after the accident, that is, the destruction of the security structure in ports caused by the emergencies will seriously affect the normal operation of the ports and the ability to adapt to and recover from the disasters.

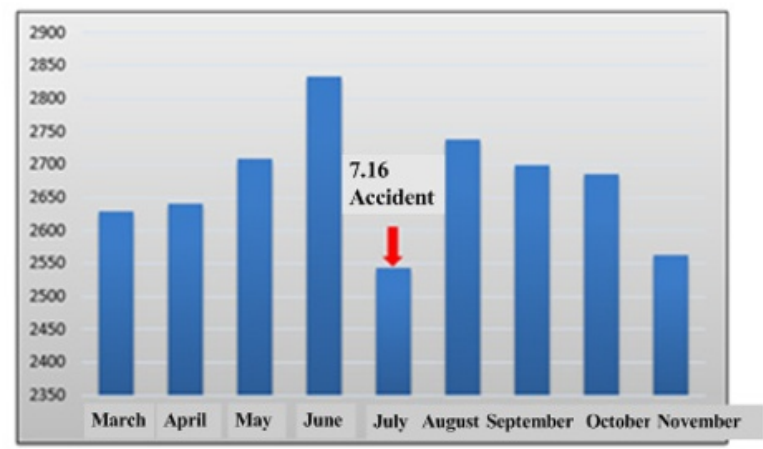

Figure 2 Throughput of Dalian Port Before and After the 7.16 Accident in 2010

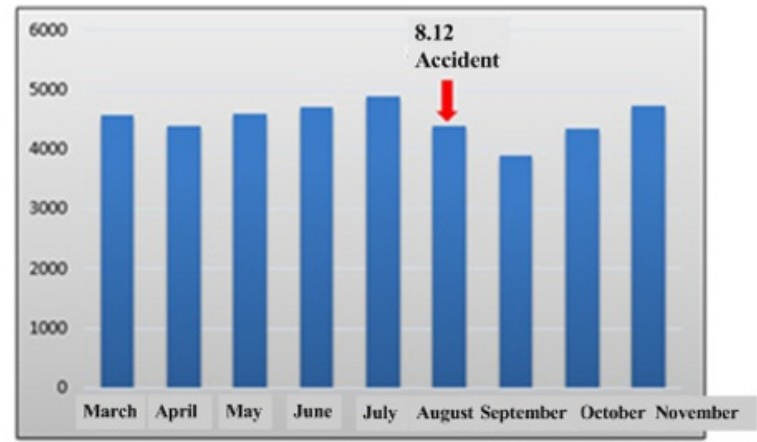

Figure 3 Throughput of Tianjin Port Before and After the 8.12 Accident in 2015

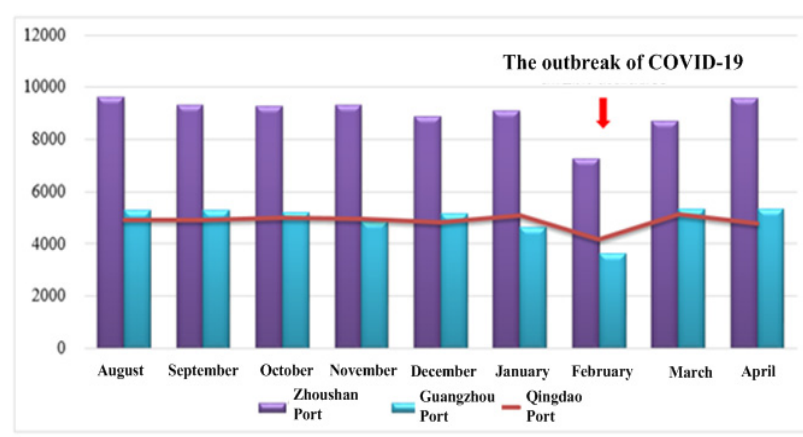

Figure 4 Throughputs of Large-Scale National Ports Before and After the Outbreak of COVID-19

As an open and complex system, the port consists of three subsystems, namely, the human, the materials and the operation system. From the analysis of the accidentcausing theories, it can be seen that once any subsystem breaks such as the human, the materials or the operation system, it will lead to the disorder of the whole complex system of the port, that is, safety accidents. Nevertheless, the problem of the port is not just limited to this extent, but also affected by the input of emergencies (such as epidemics, terrorist attacks, fire explosions, natural disasters, etc.). Taking COVID-19 as an example, when the emergency interacts with the three subsystems of the 
port, the structure of security in the port will fluctuate greatly and become unstable. Therefore, the follow-up security management in domestic ports should focus on enhancing their capacities to resist disasters or destructive changes, so as to ensure that the ports can operate stably at an acceptable level of security after their exposure to disasters. In other words, ports should have excellent safety resilience to better cope with the impact of various types of major emergencies.

\subsection{Index System of Safety Resilience in Ports}

According to the theory of the safety resilience in ports and the analysis of the triangle model of its resilience development, the port with safety resilience should reflect the characteristics of diversity, redundancy, adaptability, robustness, coordination and recovery, etc. Diversity refers to the richness of port system elements and management modes to cope with various interference forms of emergencies. Redundancy refers to the multichannel security defense in the system function and management ability of the port, which can effectively deal with the impacts and destruction of major emergencies on its infrastructure, production equipment and operation management system. Adaptability refers to the ability of port system elements and management modes to adjust flexibly according to the changes of external environment. Robustness refers to the ability of the port system to maintain basic production and operation at an acceptable level of security in an inverting environment. Coordination refers to the integration of relevant resources as much as possible in the process of port management to achieve regional joint defense and joint control. Recovery refers to the rapid restoration and reconstruction of the port system after major emergencies, which can minimize casualties and property losses. At the level of practical operation, it mainly emphasizes the safety culture of the port, the safety margin of the system, the prevention and control of accidents, risk monitoring and early warning, emergency incident management and rapid response to emergencies, and post-recovery and reconstruction after accidents, etc. On this basis, the index system is established as shown in Table 1.

Table1. Port Safety Resilience Evaluation Index System

\begin{tabular}{|c|c|c|c|}
\hline $\begin{array}{l}\text { First- } \\
\text { grade } \\
\text { indexes } \\
\text { L1 }\end{array}$ & $\begin{array}{l}\text { Second- } \\
\text { grade } \\
\text { indexes L2 }\end{array}$ & $\begin{array}{l}\text { Third-grade } \\
\text { indexes L3 }\end{array}$ & $\begin{array}{c}\text { Characteristics } \\
\text { of safety } \\
\text { resilience }\end{array}$ \\
\hline \multirow{5}{*}{$\begin{array}{l}\text { Safety } \\
\text { resilience } \\
\text { in the } \\
\text { port }\end{array}$} & \multirow{2}{*}{$\begin{array}{l}\text { Safety culture } \\
\text { L2-1 }\end{array}$} & Leadership L3-1 & \multirow{2}{*}{$\begin{array}{l}\text { Diversity, } \\
\text { robustness, } \\
\text { coordination }\end{array}$} \\
\hline & & Cohesion L3-2 & \\
\hline & & & \\
\hline & \multirow{2}{*}{$\begin{array}{l}\text { Post-recovery } \\
\text { and } \\
\text { reconstruction } \\
\text { L2-6 }\end{array}$} & $\begin{array}{l}\text { Plan formulation } \\
\text { of disaster } \\
\text { recovery L3-36 }\end{array}$ & \multirow{2}{*}{$\begin{array}{l}\text { Redundancy, } \\
\text { adaptability, } \\
\text { recovery }\end{array}$} \\
\hline & & $\begin{array}{c}\text { Production } \\
\text { material reserves } \\
\text { L3-37 }\end{array}$ & \\
\hline
\end{tabular}

\subsection{Assessment and Prediction Analysis of Safety Resilience in Ports}

- Calculation Method of Entropy Weights and Result Analysis Based on Normalization Standard Processing

If the evaluation matrix of the system consists of $\mathrm{m}$ evaluation indexes of $n$ evaluation objects, when a certain evaluation sequence $x_{1}, x_{2}, \ldots, x_{\mathrm{n}}$ is normalized by standard transformation, a new sequence shall be obtained:

$$
y_{i}=\frac{x_{i}-\bar{x}}{S}, \bar{x}=\frac{1}{n} \sum_{i=1}^{n} x_{i}, S=\sqrt{\frac{1}{n-1} \sum_{i=1}^{n}\left(x_{i}-\bar{x}\right)^{2}}
$$

According to the basic principles of information theory, information can be used as a measure of the degree of system order, while entropy is a measure of the degree of system disorder [1-2]. As is indicated, the smaller the entropy value of an index is, the greater the variation degree of its index value shall be, the more information it shall provide, and the greater the role of this index in the comprehensive evaluation shall be, and the greater its weight shall be.

If the evaluation matrix of the system consists of $\mathrm{m}$ evaluation indexes of $n$ evaluation objects, it can be obtained as:

$$
\begin{gathered}
e_{i}=-\sum_{j=1}^{n} p_{i j} \cdot \ln p_{i j} \\
p_{i j}=r_{i j} / \sum_{j=1}^{n} r_{i j} \\
W_{i}=\left(1-e_{i} / \ln (m)\right) / \sum_{i=1}^{m}\left(1-e_{i} / \ln (m)\right)
\end{gathered}
$$

In the above formula, $e_{i}$ represents the information entropy of a certain evaluation index $n_{i}, p_{i j}$ represents the probability of a certain index $r_{i j}$ in the original evaluation matrix, and $\mathrm{W}_{i}$ represents the weight of a certain evaluation index $n_{i}$.

Thus, according to the normalization standard processing and the entropy weight calculation of the sample data of safety resilience in ports, the weighting exponent of each index in the index system of safety

\begin{tabular}{|c|c|c|c|c|c|}
\hline $\begin{array}{l}\text { Second- } \\
\text { grade } \\
\text { indexes } \\
\text { L2 }\end{array}$ & $\begin{array}{c}\text { Level } \\
\text { weight } \\
\text { of } \\
\text { second- } \\
\text { grade } \\
\text { indexes }\end{array}$ & $\begin{array}{l}\text { Ranking of } \\
\text { importance } \\
\text { at this level }\end{array}$ & $\begin{array}{l}\text { Third- } \\
\text { grade } \\
\text { indexes L3 }\end{array}$ & $\begin{array}{c}\text { Level } \\
\text { weight } \\
\text { of } \\
\text { third- } \\
\text { grade } \\
\text { indexes }\end{array}$ & $\begin{array}{l}\text { Ranking of } \\
\text { importance } \\
\text { at this level }\end{array}$ \\
\hline \multirow{2}{*}{$\begin{array}{l}\text { Safety } \\
\text { culture } \\
\text { L2-1 }\end{array}$} & \multirow{2}{*}{0.154} & \multirow{2}{*}{5} & $\begin{array}{l}\text { Leadership } \\
\text { L3-1 }\end{array}$ & 0.238 & 4 \\
\hline & & & $\begin{array}{c}\text { Cohesion } \\
\text { L3-2 } \\
\end{array}$ & 0.252 & 2 \\
\hline ....... & ........ & ........ & ........ & ...... & ........ \\
\hline \multirow{2}{*}{$\begin{array}{l}\text { Post- } \\
\text { recovery } \\
\text { and } \\
\text { reconstru- } \\
\text { cttion } \\
\text { L2-6 }\end{array}$} & \multirow{2}{*}{0.121} & \multirow{2}{*}{5} & $\begin{array}{c}\text { Plan } \\
\text { formulation } \\
\text { of disaster } \\
\text { recovery } \\
\text { L3-36 }\end{array}$ & 0.167 & 5 \\
\hline & & & $\begin{array}{c}\text { Production } \\
\text { material } \\
\text { reserves } \\
\text { L3-37 }\end{array}$ & 0.234 & 1 \\
\hline
\end{tabular}
resilience in ports is analyzed and obtained, as shown in Table 2 below.

Table2. Port Safety Resilience Evaluation Index System 


\section{- Prediction Analysis of Safety Resilience}

Safety resilience can provide continuous security for ports in a constantly changing environment, so that the ports can maintain normal production and safe operation. For this reason, based on the triangle model of safety resilience development in ports and the analysis of the weight indicators of indexes at all levels of the index system of safety resilience evaluation, this paper obtains the prediction curve chart of safety resilience in ports shown in Figure 5 as follows [5-6].

As is shown in Figure 5, the abscissa is the time axis, and the ordinate represents the safety resilience level of ports. The curve can dynamically reflect the process of the decline, recovery and stability of the safety function of ports under external shocks. It is not difficult to find from the figure that different indicators have different influence weights of safety resilience in ports, and the laws of their influence on safety resilience in ports are also different. The greater the weight value of the index is, the more the impact-resistance capacity of the port system can be enhanced, and the more the speed and residence time of the safety function decline in ports can be shortened, that is, the rapid response and recovery ability of the port system can be improved. For the port system, the construction of its emergency capacity is crucial to its safety resilience. Through effective responses to emergencies and rapid disposal, the damage degree and damage scope of the accident to the port can be greatly reduced, and the rapid recovery and reconstruction and normal operation of the port after disasters can be guaranteed. The predicted trend results are basically consistent with the actual situation. This shows that the curve can predict the level of current safety resilience and the future development trajectory of ports under different index inputs to a certain extent, and then it can determine the corresponding planning strategies and action plans to strengthen the safety resilience of ports. Subsequently, through methods or means such as safety culture, safety margin, risk assessment, monitoring and early warning, emergency management and recovery and reconstruction, the improvement of urban safety resilience can be targeted to improve the response of the system function in ports to external shocks.

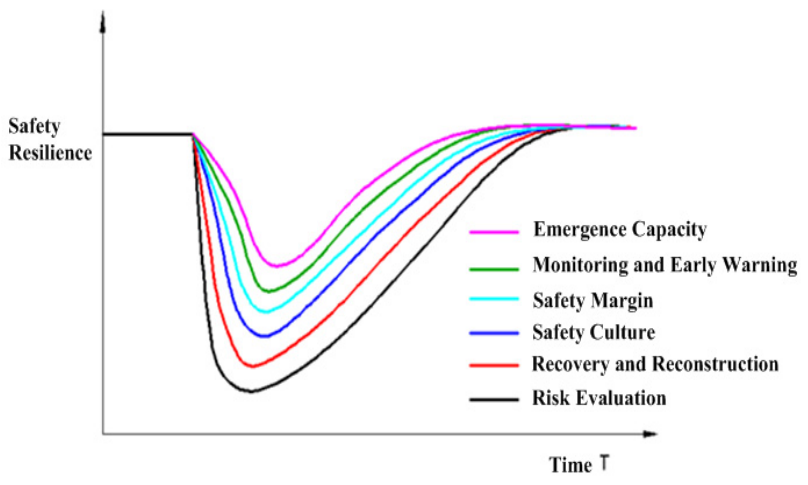

Figure 5 Prediction Curve Graph of Safety Resilience in Ports

\section{Conclusion}

This paper studied the issues of port safety under major emergencies. For the first time, the theory of safety resilience was introduced into the field of ports. Aiming at the demands for quantitative evaluation of safety resilience in ports, the system of evaluation indexes of safety resilience in ports was established, and the influence weight value of each index on safety resilience and the prediction curve of safety resilience in ports were determined. The research conclusions are as follows:

First, based on the theoretical connotation of safety resilience, the triangular model of safety resilience development in ports was constructed. The model took the disaster system, the disaster bearing system and the safety resilience management as three edges, and connected each edge through the response process of port planning and construction, operation and maintenance, event responses and recovery and reconstruction, which can reflect the management demands for the safety resilience of the whole life cycle of the port. Secondly, an index system of safety resilience in ports was constructed with security culture, security margin, risk assessment, monitoring and early warning, emergency management and recovery and reconstruction as its core elements, which included 6 secondary indexes and 37 tertiary indexes. Considering the uncertainty and information variability of the evaluation of safety resilience, a model of entropy weight calculation based on normalization standard processing was established to obtain the weight level and hierarchical importance ranking of the above indicators, which has important guiding significance for the status analysis and the expected planning of safety resilience. Thirdly, based on the evaluation results of the safety resilience, the safety resilience curve of the port was predicted, and the trend results were basically consistent with the actual situation, indicating that the method is feasible with strong application value.

In this paper, the resilience theory was applied to port security, and the evaluation system was established while calculating the index weights. However, there are still urgent theoretical and practical demands for the research and discussion of safety resilience in ports, and further exploration and researches can be carried out in the following aspects. The indexes of resilience factors in the system of evaluation indexes can be applied to further study to optimize the measurement, and deeper research still needs be conducted on how to coordinate and maximize the effects of safety resilience management in ports under major emergencies. Through data driving, safety production, safety resilience, integrated management theory and engineering philosophy, the interaction and organic integration of technical mechanism should be studied to explore the development laws of the resilience of safety production. By means of data analysis and deep learning, the resilience of safe production and the relevant policies can be constantly improved, so as to realize the optimal management of port safety. 


\section{Acknowledgment}

This research is supported by the special fund for the basic research business of the central public welfare research institutes (TKS 20200320) and 2020 key science and technology projects of transportation industry (2020MS3-097).

\section{References}

1. The State Council of the CPC Central Committee. "Outline on Building China's Strength in Transportation," Railway Quality Control, 2019, vol. 47, pp. 1-4.

2. Ministry of transport. "Guidance on building a worldclass port," China Water Transport, 2019, vol.12, pp. 19-22.

3. C. S. Holling. "Engineering resilience versus ecological resilience," Engineering Within Ecology Constraints, 1996.

4. T. Mcdaniels, S. Chang and D. Cole, "Fostering resilience to extreme events within infrastructure systems: characterizing decision contents for mitigation and adaption," Global Environmental Change,2008, vol.18, pp. 310-318.

5. UNISDR. "2009 UNISDR terminology on disaster risk reduction," Abyadh, 2009, vol.8, pp. 95-105.

6. Y. W. Shao and J. Xu, "Understanding urban resilience: a conceptual analysis based on integrated international literature review," Urban Planning International,2015, vol.30, pp. 48-54.

7. Q. L. Bing, X. Li and Y. Luo, "Urban Disaster Prevention Plan with Resilient City Theory," Planners,2017, vol. 33, pp. 12-17.

8. L. Zou, "The concept of resilience in urban disaster prevention,” Beijing Planning Review,2018, vol. 2, pp. 18-21.

9. Y. Zheng, "Promoting urban adaptation planning and building resilient cities - the cases and enlightenments of developed countries," World Environment,2013, vol. 6, pp. 50-53.

10. X.D. Guo, J. Y. Su amd Z.T. Wang, "Urban safety and disaster prevention under the perspective of resilience theory," Shanghai Urban Planning Review,2016, vol. 1, pp. 41-44.

11. G.L. Lin, Y. C. Li and L. J. Qu, "Evaluation of Emergency Response Capability of Port Safety Accidents," Safety and Environmental Engineering,2020, vol.5, pp. 160-164.

12. J. X. Liu, "Research on hidden danger classification investigation and control for port equipment based on risk management," Tianjin university of technology, 2019.

13. C. Huang, Y. Bai and S. F. Zhan, "Evaluation model of safety performance of general cargo port enterprises based on fuzzy theory," Journal of Waterway and Harbor,2019, vol. 40, pp. 117-122.
14. J. Zhang, X. D. Li and Z. H. Zhang, "Support Capacity Analysis of Commercial Seaport Infrastructures from the Perspective of National Security," Journal of Tianjin University(Science and Technology), 2020, vol.9, pp. 83-90.

15. W. C. Fan, "The development trend of safe and resilient cities," Labour Protection,2020, vol.3, pp. 20-23.

16. W. C. Fan, Y. Liu and W. G. Weng, "Triangular framework and " $4+1$ " methodology for public security science and technology," Science \& Technology Review,2009, vol.27, pp. 3.

17. Y. Shi, B. Meng and N. Lu, "Risk Assessment and Analysis of General Aviation Operation Based on AHP and Entropy Weight," Journal of Shanghai University of Engineering Science, 2019, vol.33, pp. 47-51

18. J. N. Sun and G. P. Ren, "Study and Application on the Entropy method for Determination of Weight of evaluating indicators in Fuzzy Synthetic Evaluation for Water Quality Assessment," Acta Scientiae Circumstantiae, 2005, vol. 25, pp. 552-556.

19. R. Q. Li, H. Huang and R. Zhou, "Resilience curve modeling of urban safety resilience," Journal of Tsinghua university (Science \& technology) , 2020, vol.60, pp. 1-8.

20. J. M. Grove, R. R. Chowdhury and D. Childers, "Codesign, co-production, and dissemination of socialecological knowledge to promote sustainability and resilience: urban experiences from the U.S," Global Land Project News, 2015, vol.11, pp. 6-11.

21. C. S. Holling, "Resilience and Stability of Ecological Systems," Annual Review of Ecology and Systematics, 1973, pp. 1-23.

22. S. R. Trammell and R. D. Wright, "Integrating Risk Assessment Into Management Systems," Electronics Manufacturing Technology Symposium, 1999, pp. 156-159.

23. E. Hollnagel and D. Woods, "Epilogue: Resilience engineering -concepts and precepts," Aldershot: Ashgate Publishing, 2006, pp. 347-58.

24. A. W. Righi, T. A. Saurin and P. Wachs, "A systematic literature review of resilience engineering: research areas and a research agenda proposal," Reliability Engineering and System Safety, 2015, vol.141, pp. 142-152.

25. W. C. Fan, "Thoughts and suggestions on building a smart and resilient City," Informatization of China's Construction, 2015 vol. 21, pp. 20-21.

26. L. Huang, C. Wu and M. Yang, "Application of toughness theory in Safety Science," Chinese Journal of Safety Science, 2017, vol. 27, pp. 1-6.

27. Y. Luo, X. F. Huang and M. Xu, "Discussion on the development and trend of scientific management of work safety," Safety production science and technology in China, 2016, vol. 12, pp. 5-11.

28. Y. Li, G. F. Zhai and F. M. Gu, "Review on quantitative evaluation methods of urban 
infrastructure resilience," Research on urban development, 2016, vol. 23, pp. 113-122.

29. K. C. Desouza and T. H. Flanery. "Designing, planning, and managing resilient cities: A conceptual framework," Cities, 2013, vol. 35, pp. 89-99.

30. A. K. Jha, T. W. Miner and Z. Stanton-Geddes, "Building Urban Resilience: Principles, Tools, and Practice," World Bank Publications, 2013.

31. S. L. Cutter, L. Barnes and M. Berry, "A place-based model for understanding community resilience to natural disasters," Global Environmental Change, 2008, vol.18, pp. 0-606.

32. S. L. Cutter, C. G. Burton and C. T. Emrich, "Disaster resilience indicators for benchmarking baseline conditions," Journal of Homeland Security \& Emergency Management, 2010, vol.7, pp. 1271-1283.

33. J. D. F. António, P. João and M. Malta, "Improving urban ecosystems resilience at a city level the Coimbra case study," Energy Procedia, 2013, vol. 40, pp. 6-14. 